\title{
Novel real-time alignment and calibration of the LHCb detector in Run II
}

\section{Giulio Dujany ${ }^{* \dagger}$}

The University of Manchester

E-mail: giulio.dujany@cern.ch

Stable, precise spatial alignment and PID calibration are necessary to achieve optimal detector performance. During Run II, LHCb will have a new real-time detector alignment and calibration to allow equivalent performance in the online and offline reconstruction to be reached. This offers the opportunity to optimise the event selection by applying stronger constraints, and to use hadronic particle identification at the trigger level and even to perform physics analysis directly on the trigger output. The computing time constraints are met through the use of a new dedicated framework using the multi-core farm infrastructure for the trigger. The motivation for a real-time alignment and calibration of the LHCb detector is discussed both from the operative and physics performance point of view. Specific challenges of this novel configuration are discussed, as well as the designed framework and its performance.

24th International Workshop on Vertex Detector -VERTEX2015-

\section{1-5 June 2015}

Santa Fe, New Mexico, USA

\footnotetext{
${ }^{*}$ Speaker.

$\dagger$ on behalf of the LHCb collaboration.
} 


\section{Introduction}

The LHCb detector $[1,2]$ is a single-arm forward spectrometer covering the pseudorapidity range $2<\eta<5$, designed for the study of particles containing $b$ or $c$ quarks. The detector includes a high-precision tracking system, which provides a measurement of momentum of charged particles, with a relative uncertainty that varies from $0.5 \%$ at low momentum to $1.0 \%$ at $200 \mathrm{GeV} / c$. The minimum distance of a track to a primary vertex, the impact parameter, is measured with a resolution of $\left(15+29 / p_{T}\right) \mu \mathrm{m}$, where $p_{T}$ is the component of the momentum transverse to the beam in $\mathrm{GeV} / c$. The tracking system consists of a silicon-strip vertex detector (VELO) surrounding the $p p$ interaction region [3], a large-area silicon-strip detector located upstream of a dipole magnet with a bending power of about $4 \mathrm{Tm}$, and three stations of silicon-strip detectors and straw drift tubes [5] placed downstream of the magnet. Different types of charged hadrons are distinguished using information from two ring-imaging Cherenkov detectors (RICHs) [6]. Photons, electrons and hadrons are identified by a calorimeter system consisting of scintillating-pad and preshower detectors, an electromagnetic calorimeter and a hadronic calorimeter. Muons are identified by a system composed of alternating layers of iron and multiwire proportional chambers [7]. The online event selection is performed by a trigger [8], which consists of a level zero hardware stage (L0), based on information from the calorimeter and muon stations, followed by a two software stages. The first software stage (HLT1) performs a track fit using the information from the VELO and the tracking stations while the second stage (HLT2) applies a full event reconstruction with the information from all subdetectors.

The spatial alignment of a detector and the accurate calibration of its subcomponents are essential elements to achieve the best physics performance. The correct alignment of the VELO is needed to identify primary vertices and secondary vertices from the decay of particles containing $b$ or $c$ quarks while a misalignment of the all tracking system would degrade the momentum and mass resolution. Figure 1 shows how an improved alignment greatly enhances the $\Upsilon$ mass resolution from $92 \mathrm{MeV} / c^{2}$ with the first alignment to $49 \mathrm{MeV} / c^{2}$ with the improved one.
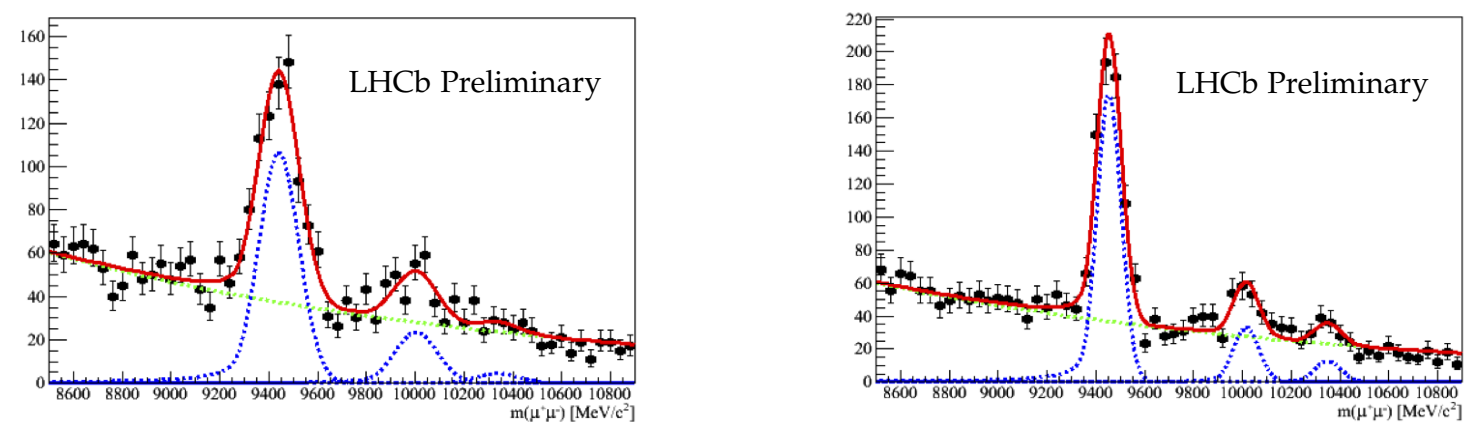

Figure 1: Invariant mass distribution for $\Upsilon \rightarrow \mu \mu$. The mass resolution is $92 \mathrm{MeV} / c^{2}$ with the first alignment (left) and is enhanced to $49 \mathrm{MeV} / c^{2}$ with an improved alignment (right).

An exclusive selection using hadron identification criteria relies on the complete calibration of the ring-imaging Cherenkov detectors. Figure 2 shows the effect in the $B^{0} \rightarrow h^{+} h^{-}$mass spectrum of hadron identification criteria: the ratio between the signal, $B^{0} \rightarrow \pi^{+} \pi^{-}$, and the combinato- 
rial background increases by approximately a factor two and the ratio between the signal and the favoured $B^{0} \rightarrow K \pi$, increases by a factor 35 . Is it thus clear that using a real-time alignment and
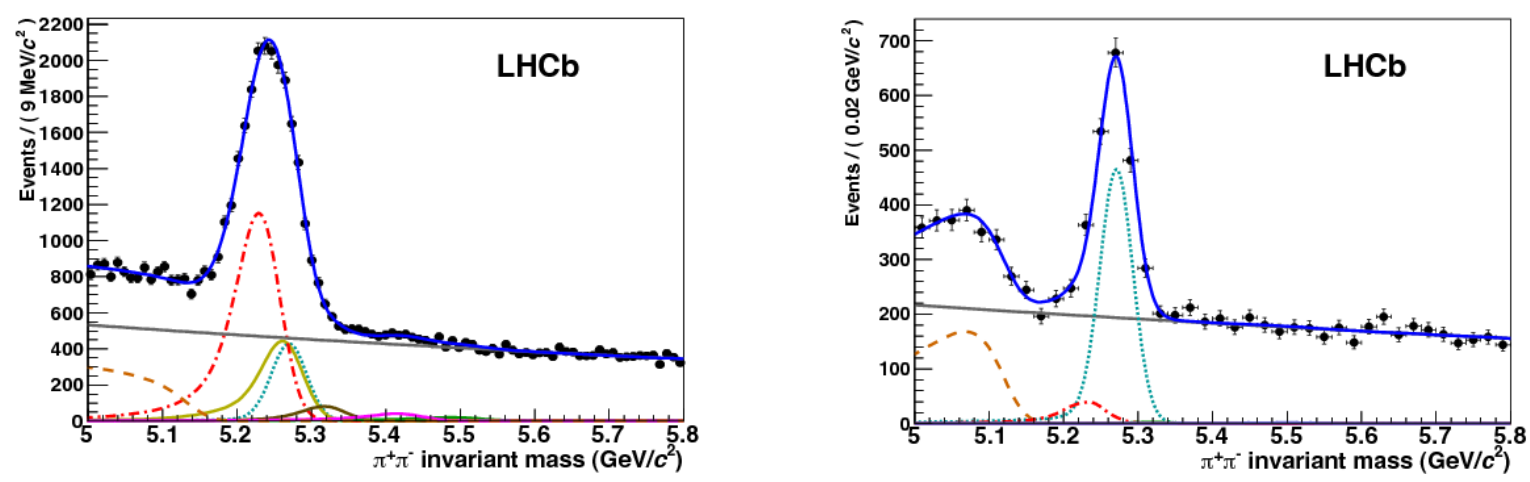

Figure 2: Invariant mass distribution for $B^{0} \rightarrow h^{+} h^{-}$decays [9] in the LHCb data before the use of the RICH information (left), and after applying RICH particle identification (right). The signal under study is the decay $B^{0} \rightarrow \pi^{+} \pi^{-}$, represented by the turquoise dotted line. The contributions from different $b$ hadron decay modes $\left(B^{0} \rightarrow K \pi\right.$ red dashed-dotted line, $B^{0} \rightarrow 3$-bodies orange dashed-dashed line, $B_{s} \rightarrow K K$ yellow line, $B_{s} \rightarrow K \pi$ brown line, $\Lambda_{b} \rightarrow p K$ purple line, $\Lambda_{b} \rightarrow p \pi$ green line), are eliminated by positive identification of pions, kaons and protons. Only the signal and two background contributions remain visible after applying hadron identification requirements. The grey solid line is the combinatorial background.

calibration during the trigger selection allows a higher signal purity and a more effective selection on the channels interesting to pursue LHCb's physics program without increasing the overall trigger rate. This, however, presents an unprecedented challenge as it requires to align more than 1700 detector components and compute almost 2000 calibration constants on the fly.

\section{Expected alignment variations from Run I}

The VELO is made of two halves that during the data taking are at approximately $8 \mathrm{~mm}$ away from the nominal beam position. For the safety of the detector during the beam injection at the beginning of a fill the halves are retracted by $29 \mathrm{~mm}$; when stable beam is declared the VELO is closed around the beam.

The VELO halves are moved using stepper motors and their position is read from resolvers mounted on the motor axes with an accuracy better than $10 \mu \mathrm{m}$. An automated closure procedure positions the VELO halves around the beams using the response of the hardware and the measured positions of the beams. By considering the two independent beam profiles compiled by each half, the VELO is observed to close symmetrically around the beams to an accuracy of better than $4 \mu \mathrm{m}$. As the VELO is closed for each fill, its alignment may change with the same frequency.

Figure 3 shows for a subsample of the Run I dataset the variation of the misalignment between the two halves in the horizontal direction perpendicular to the beam. It ca be estimated by taking the difference of the positions of the primary vertices reconstructed separately with tracks in only one half of the VELO. In a perfectly aligned detector the mean of this difference should be zero. The average variation in Run I is $\sim 4 \mu \mathrm{m}$ while the maximum variation is $O(10 \mu \mathrm{m})$, which is more than the $O(2 \mu \mathrm{m})$ precision of the track based software alignment procedure [3]. 


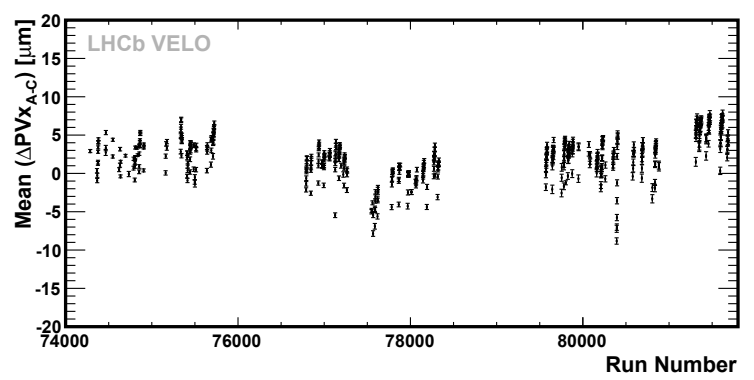

Figure 3: Misalignment between the two VELO halves along the main movement direction for the different runs, evaluated by fitting the primary vertices separately with tracks in each half of the VELO. The run numbers shown here span the period of the last four months of operations in 2010 .

For the other components of the tracking system, in addition to the variation due to hardware intervention, some variation over time was observed, partially correlated to the magnet polarity which is reversed periodically. During Run I a new tracking alignment has been evaluated after each magnet polarity switch or technical stop. This strategy allowed to have a momentum scale and resolution stable with time as shown in Figure 4.
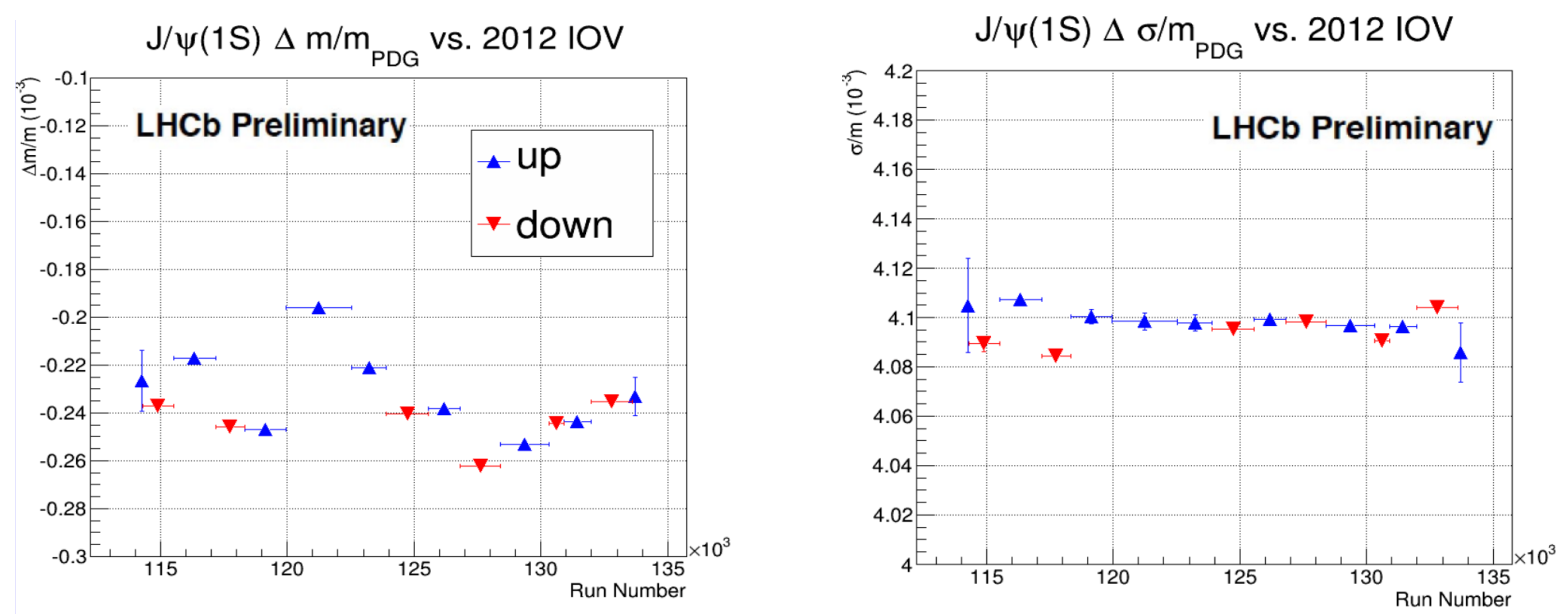

Figure 4: Time evolution of the relative variation of the difference between the measured mass of the $J / \Psi(1 \mathrm{~S})$ and the nominal one from [4] (left) and relative variation of the mass resolution at the $J / \Psi(1 \mathrm{~S})$ mass (right). Each point corresponds to data taken with a different tracking alignment; the blue up-triangles and red down-triangles correspond to opposite magnet polarities.

\section{Trigger strategy for Run II}

In Run I the rate of collisions was $15 \mathrm{MHz}$ and will double in Run II while the output rate of events saved on disk will change from $5 \mathrm{kHz}$ in Run I to $12.5 \mathrm{KHz}$ in Run II. In Run I the event reconstruction performed online by the trigger was simpler and quicker than the one performed offline on triggered events in order to meet time contraints; the final detector calibration and an improved alignment were obtained offline on triggered data and the data used for most of the physics results was processed at the end of the year using the latest constants.

During 2012 LHC spent only approximately $30 \%$ of its time in stable running due to e.g. planned technical stops, machine developpement phases and the time between data taking fills needed for the ramping of the LHC dipole magnets. In order to optimise the usage of the event 
filter farm where the software trigger is run, the farm nodes were equipped with local storage space and $25 \%$ of L0 output was buffered into the nodes and processed during the LHC downtime.

In Run II this deferral strategy is exploited even further as shown in Figure 5; after L0 and HLT1 all the selected events are buffered on disk allowing to have more time to process a single event (150 ms/event in Run I, 350 ms/event in Run II). The automatic calibration and alignment is performed in the trigger farm within a few minutes. The same offline reconstruction is run in HLT2 thanks to this larger time budget together with the reduced time requested by the improved track reconstruction.

When the new alignment and calibration are available, and a significant variation is observed, a change of run is triggered. The new constants are updated for the new run to be used online by the two stages of the software trigger, and offline, for every further reconstruction and selection. The events collected in the previous run, during the automatic alignment and calibration processing, are still reconstructed in HLT2 and offline with the previous constants for consistency with HLT1. As exception the RICH calibration constants are updated for each run after HLT1 both online, in HLT2, and offline as the previous trigger stages do not rely on hadron identification requirements.

This strategy has several advantages: firstly it minimises the difference between the online and offline performance, allowing to use a more effective trigger selection that can take advantage of the hadron identification information. For example charm physics is limited by trigger output rate contraints; using hadron identification in the trigger allows to have a higher selection efficiency and purity for the doubly Cabibbo suppressed modes and, at the same time, satisfy the output rate constraints by pre-scaling the more abundant Cabibbo favourite modes. Secondly it ensures the stability of the alignment quality and hence of the physics performance. Finally, as in Run II the last level of the trigger performs the same reconstruction as the one performed offline, it will be possible to run some physics analyses, with the same offline performance, directly on the output of the trigger using the special stream of data known as Turbo stream [10]. This approach has the advantage that, for some events, it will be possible to save only the information on the signal candidate tracks $(\sim 5 \mathrm{kB}$ per event) instead of all the electronic signals recorded from the detector ( $\sim 70 \mathrm{kB}$ per event). This decrease of more than one order of magnitude of the event size will allow a higher selection rate, for example in the charm analyses that during Run I used trigger lines which were pre-scaled due to output rate requirements. It is obvious that the real-time alignment and calibration becomes essential when the raw event is not saved.

\section{The real-time alignment procedure}

Two main kinds of tasks are defined: alignment tasks and calibration tasks. The real-time evaluation of the alignment and the calibration uses for each task a dedicated data sample selected by HLT1, and is performed at regular intervals: either at the beginning of the run, fill, or less frequently depending on the task. They are performed in a few minutes using the nodes of the trigger farm and the alignment or calibration constants are updated only if the difference from the previous values is significant, to ignore a random fluctuation due to the different input data sample used. 
LHCb 2015 Trigger Diagram

\section{$40 \mathrm{MHz}$ bunch crossing rate}
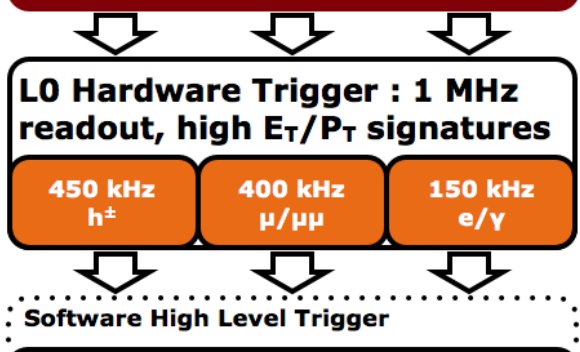

Partial event reconstruction, select displaced tracks/vertices and dimuons

Buffer events to disk, perform online detector calibration and alignment : L

Full offline-like event selection, mixture of inclusive and exclusive triggers

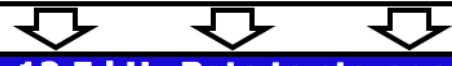

$12.5 \mathrm{kHz}$ Rate to storage
Figure 5: Trigger strategy for Run II: after the hardware stage and a first software stage based on a partial reconstruction, the selected events are buffered on disk while the real-time calibration and alignment are performed. The second stage of the software trigger performs the same reconstruction performed offline using the same calibration and alignment.

\subsection{Tracking alignment}

The tracking alignment is based on an iterative procedure where the residuals of a Kalman track fit are minimised. The magnetic field and material effects are taken into account and information from vertices and particle masses can also be used as constraints to avoid global distortions $[11,12]$.

Given a set of tracks reconstructed using the alignment parameters $\alpha_{0}$, the new set of alignment constants can be found solving the system of equations

$$
\alpha=\alpha_{0}-\left.\left.\left(\frac{d^{2} \chi^{2}}{d \alpha^{2}}\right)^{-1}\right|_{\alpha_{0}} \frac{d \chi^{2}}{d \alpha}\right|_{\alpha_{0}}
$$

where the derivatives of the total $\chi^{2}$ with respect to the alignment parameters are obtained by summing the contributions from all the tracks:

$$
\begin{aligned}
\frac{d \chi^{2}}{d \alpha} & =2 \sum_{\text {tracks }}{\frac{d r^{T}}{d \alpha}}^{T} V^{-1} r \\
\frac{d^{2} \chi^{2}}{d \alpha^{2}} & =2 \sum_{\text {tracks }} \frac{d r^{T}}{d \alpha} V^{-1} R V^{-1} \frac{d r}{d \alpha} .
\end{aligned}
$$

Here $V$ is the covariance matrix of the measurement coordinates, $r$ is the track residuals (the distance between the hit position and the track intercept point), and $R$ is the covariance matrix of the residuals. It is assumed that the $\chi^{2}$ has been minimised with respect to the track parameters for the alignment $\alpha_{0}$.

The computation of the $\chi^{2}$ derivatives can be parallelised by reconstructing the tracks and computing part of the sum over different events on different nodes. The partial sums can then be 
added and Equation (4.1) minimised on a single node. For this reason two different alignment tasks are defined.

- The analyser performs the track reconstruction based on the input set of alignment constants and evaluates the partial of the sums from Equation (4.2). Many instances run in parallel within the $\sim 1700$ nodes of the HLT farm. In order not to compete with the HLT1 processes just one instance is run per node.

- The iterator collects the output of all the analysers and minimises Equation (4.1).

The behaviour of both the analyser and the iterator are defined by the finite state machine in Figure 6. After the initial configuration, the analysers read the initial alignment constants and run on the events assigned to them. The partial sums computed by the analysers are written on a fixed location of a shared file system when all the events have been analysed. The iterator then reads the output of the analysers, combines them and computes a new set of alignment constants. The analysers then start a new iteration of the alignment procedure starting from the new alignment constants. The iterations continue until the difference of the $\chi^{2}$ between two successive iterations falls below a threshold. The reason for this is that the change in the total $\chi^{2}$ is equivalent to the significance of the alignment correction:

$$
\Delta \chi^{2}=-\left.\left(\alpha-\alpha_{0}\right)^{T} \frac{d \chi^{2}}{d \alpha}\right|_{\alpha_{0}}=-\left(\alpha-\alpha_{0}\right)^{T} \operatorname{Cov}\left(\alpha_{0}\right)^{-1}\left(\alpha-\alpha_{0}\right)
$$

where $\operatorname{Cov}\left(\alpha_{0}\right)=\left.\left(\frac{d^{2} \chi^{2}}{d \alpha^{2}}\right)^{-1}\right|_{\alpha_{0}}$ is the covariance matrix for the alignment parameters.

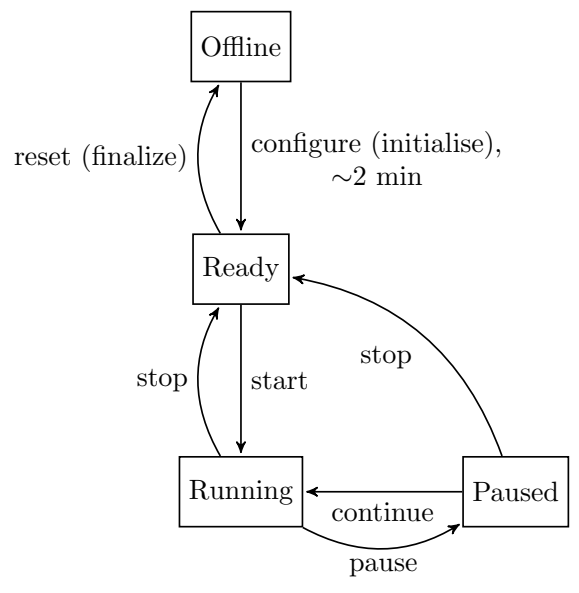

Figure 6: Finite state machine which defines the behaviour of the alignment tasks.

The alignment procedure for the different components of the tracking system will be evaluated at the beginning of each fill, but from Run I experience the frequency of expected updates is different.

The alignment of the VELO is evaluated first and, as the alignment of the VELO can change for each fill, in case a significant variation of alignment parameters is found, a change of run is triggered and the new alignment constants are used from the following run. An update of the VELO alignment parameters is expected often but not for each fill. 
The alignment of the tracker is run after the alignment of the VELO. These alignment constants are expected to change every few weeks and, if a significant variation is found, the new alignment parameters are applied to the following fill.

The alignment procedure of the muon stations is also run at the beginning of each fill after the tracker's one but only serves as monitoring, as its alignment is not expected to vary except in case of hardware intervention.

\subsection{Rich Mirror alignment}

Both RICH detectors have two sets of mirrors: photons are reflected off a primary mirror onto a secondary mirror, from where they are deflected out of the LHCb acceptance onto the photon detection plane.

The RICH mirror alignment follows the same general procedure of the tracking alignment: there is a task performed in parallel by the analysers while the computation of the alignment constants is performed by the iterator on a single node. The RICH mirror alignment relies on the fact that a misalignment of the mirrors causes the Cherenkov ring on the photodetector plane to be shifted with respect to its expected position. The projected track coordinate is not at the centre of the ring, thus the Cherenkov opening angle $(\Theta)$ varies as a function of the azimuthal angle $(\phi)$ in the photodetector plane:

$$
\Delta \Theta=\Theta-\Theta_{0}=T_{x} \cos (\phi)+T_{y} \sin (\phi),
$$

where $T_{x}$ and $T_{y}$ are the components of the misalignment of the projected track coordinate with respect to the expected position and $\Theta_{0}$ is the Cherenkov angle calculated from the momentum of the track and the refractive index of the radiator [6].

The analysers perform the photon reconstruction and fill histograms of the $\Delta \Theta(\phi)$ distribution for each pair of mirrors on different events. The iterator collects all the histograms and combines them. It fits the combined histogram using Equation (4.4) and extracts the alignment constants. The procedure is iterated until the variations are below a threshold. Figure 7 shows for one mirror the distribution of $\Delta \Theta$ as a function of $\phi$ before and after the mirror alignment.
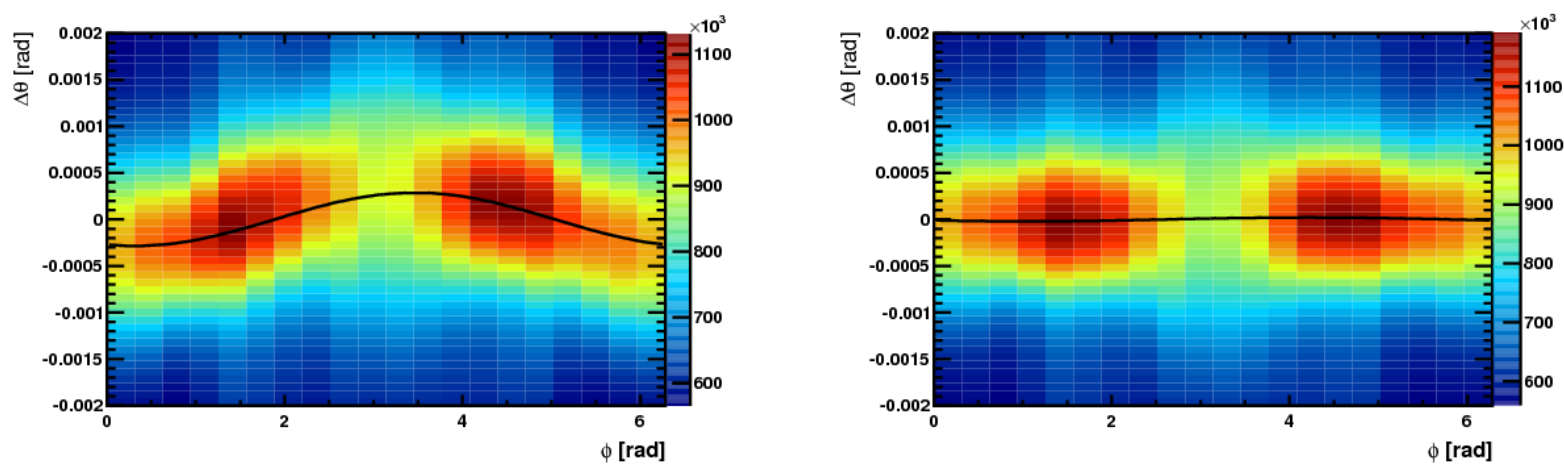

Figure 7: Difference between the measured and expected Cherenkov angle $\Delta \Theta$ as a function of the azimutal angle $\phi$ before (left) and after (right) the mirror alignment for one mirror [6]. 


\section{The real-time calibration procedure}

\subsection{RICH calibration}

The RICH automatic calibration consists of calibrating the RICH refractive index and the Hybrid Photon Detectors (HPDs) images. Both these calibrations are evaluated and updated every run.

The refractive index of the gas radiators depends on the ambient temperature and pressure, and the exact composition of the gas mixture and so can change in time. These quantities are monitored to compute an expected refractive index, but this does not have a high enough precision and needs to be corrected. The distribution of the difference between the reconstructed and expected Cherenkov angle is fitted to extract the scale factor to correct the expected refractive index.

HPDs are used to detect Cherenkov photons. They consist of vacuum tubes separated from the radiator gas by a quartz window and a photocathode. The photoelectrons produced are focused onto a silicon pixel array using an accelerating voltage [6]. The anode images are affected by magnetic and electric fields, and so are cleaned and a Sobel filter [13] is used to detect the edges. Figure 8 shows the anode images before and after this process. The evaluation of the new calibration constants does not require an iterative procedure and can be obtained by fitting the relevant distribution on a single node.
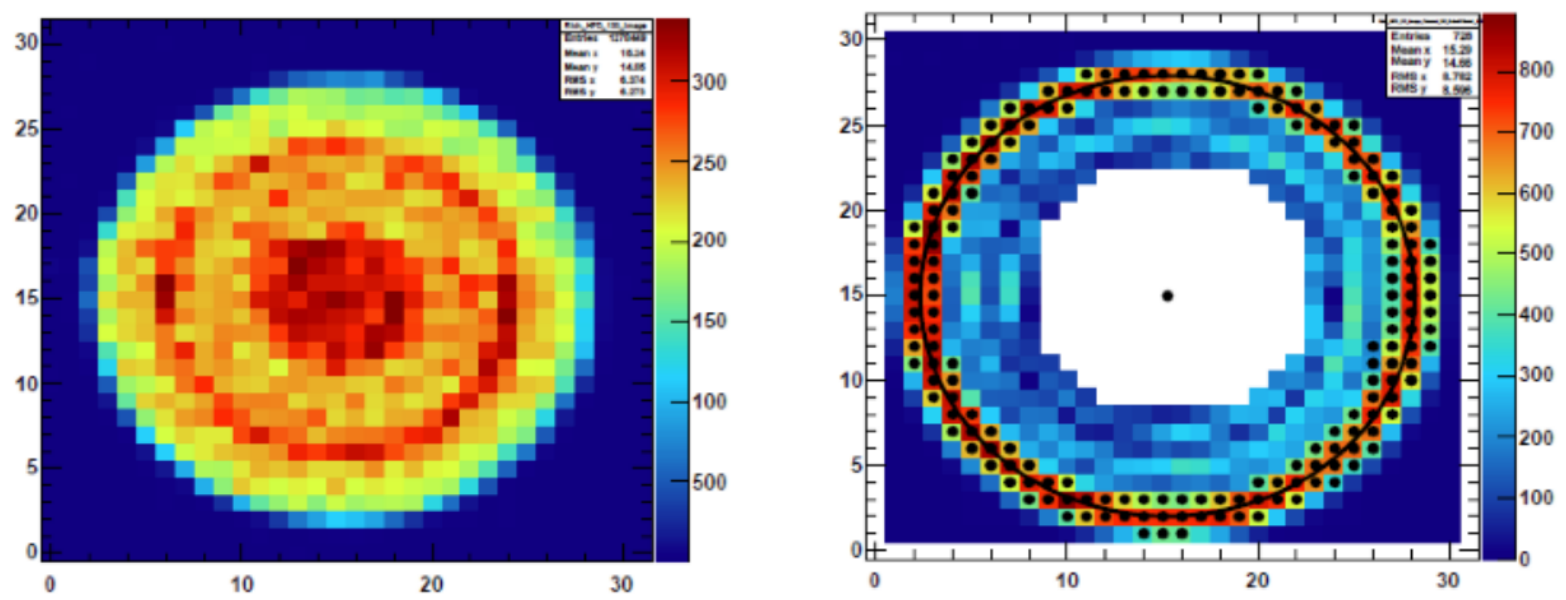

Figure 8: RICH HPD anode images, before (left) and after (right) the cleaning and applying the Sobel filter.

\subsection{Global time alignment of the Outer Tracker}

In the straw tubes that compose the Outer Tracker, the drift time measured may be different from the time estimated from the distance of the track to the wire. This is mainly due to the difference between the collision time and the LHCb clock and is common to all modules. A delay in the electronic readout, different for each module, may be partially responsible for this difference, but its contribution is small in comparison and constant in time, thus it can be calibrated offline. The automatic procedure is performed every fill and computes a single parameter which accounts for the global time alignment by fitting on a single node the distribution of the difference between the measured and the estimated time (Figure 9). 


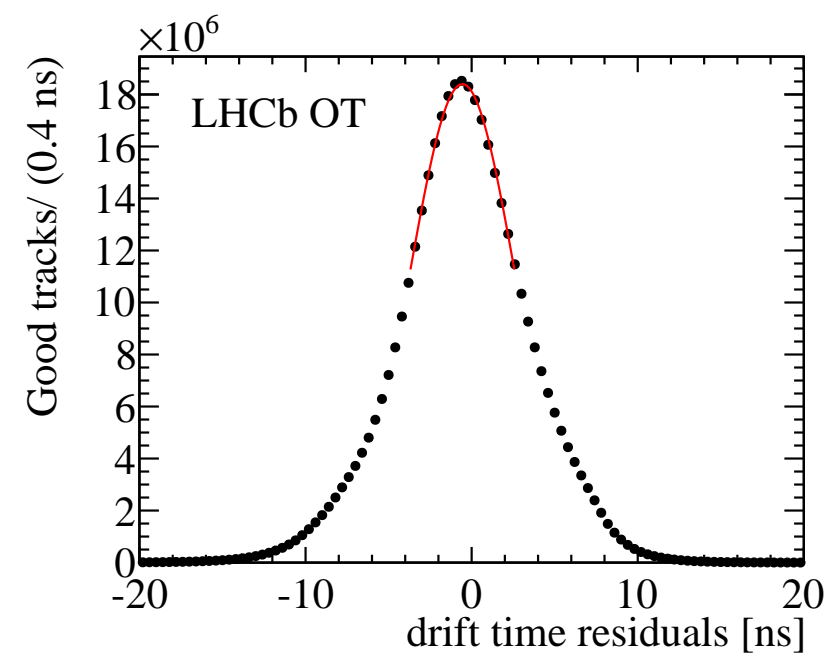

Figure 9: Fit to the distribution of the drift-time residuals of Outer Tracker hits used to estimate the global time shift between the collision time and the $\mathrm{LHCb}$ clock.

\subsection{Calorimeter calibration}

In order to have a constant L0 rate the calorimeter gain should not change with time. The variation of the gain can be estimated by the variation of the relative occupancy. The occupancy for a cell is defined as the fraction of events in which the ADC output is above a threshold. The ratio of the occupancies with respect to a reference sample is proportional to the changes in gain. A relative calibration is performed online on a single node for each fill using this occupancy method and when needed the high voltage is changed accordingly.

An absolute calibration can be obtained with the $\pi^{0}$ method: the reconstructed $\pi^{0}$ mass can be determined for each cell by fitting di-photon mass distributions where one of the photon has the cell as the seed. The calibration coefficients can be tuned to constrain the reconstructed $\pi^{0}$ mass to the nominal one. This fine calibration requires an iterative procedure and it is run on the HLT farm similarly to the tracking or RICH mirror alignment. This second method takes several hours to run and will be performed a few times per year i.e. during technical stops.

\section{First tracking alignments in Run II}

At the beginning of the Run II data taking the commissioning of the real-time alignment was done on the new data. Figures 10 and 11 show the convergence of the firsts automatic alignment for the VELO and the tracker starting from the 2012 alignment. For the VELO the misalignment is small and compatible with the one expected between successive fills. While, for the Inner Tracker a large misalignment of a couple of millimeters was expected due to a mechanical intervention during the long shutdown. Figure 11 shows the convergence of the automatic alignment procedure when starting from such large misalignment; during normal operations the variations are expected to be smaller and the procedure is expected to converge in 2-3 iterations.

\section{Conclusion}

In Run II the new scheme for the software trigger at $\mathrm{LHCb}$ allows the alignment and calibration to be performed in real time. A dedicated framework has been put in place to parallelise the 

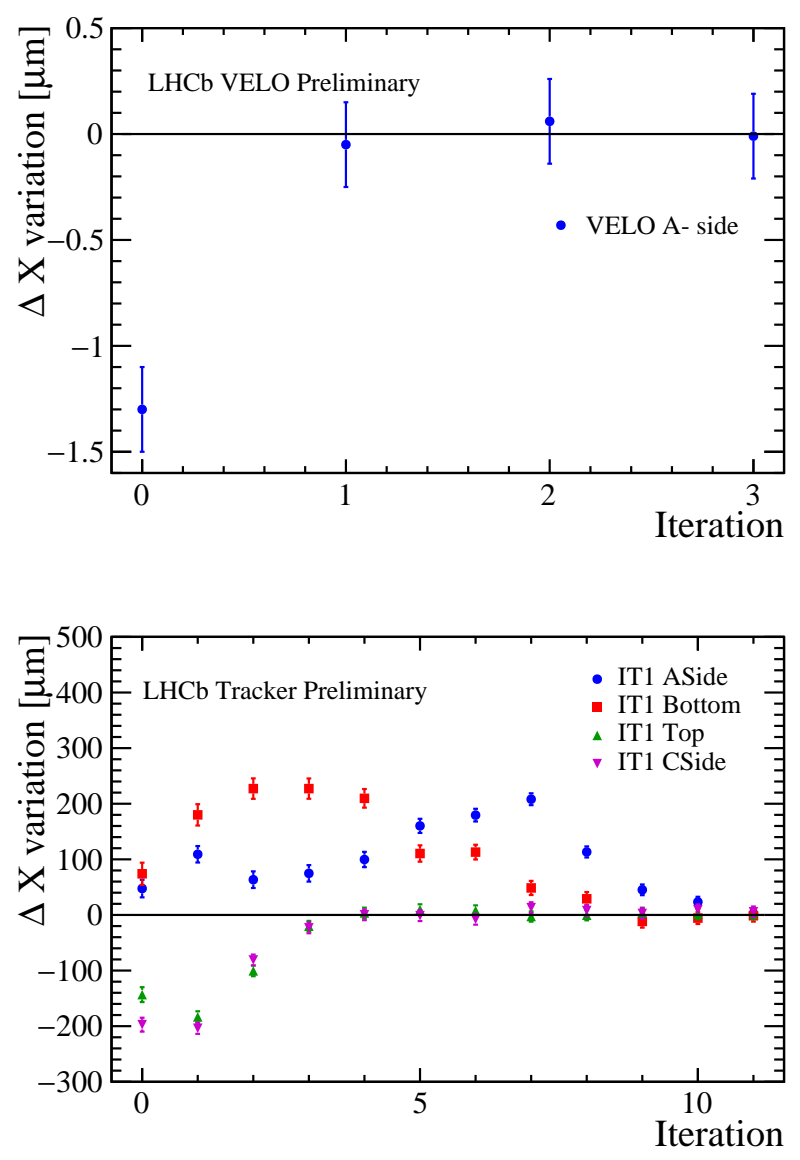

Figure 10: Convergence of the VELO alignment obtained on fill 3819 starting from 2012 VELO alignment. Each point shows the variation of the VELO left side $\mathrm{x}$-translation with respect to the previous iteration.

Figure 11: Convergence of the tracker alignment obtained on fill 3835 starting from 2012 tracker alignment. Each point shows the variation of the alignment parameter with respect to the previous iteration.

alignment and calibration tasks on the multi-core farm infrastructure used for the trigger in order to meet the computing time constraints. Data collected at the start of the fill are processed in a few minutes and the output is used to update the alignment, while the RICH calibration constants are evaluated for each run. The same framework is used to perform finer calibration less frequently and to monitor the alignment quality of various subdetector. This procedure allows a more stable alignment quality, more effective trigger selections and online-offline consistency thanks also to the same online-offline reconstruction. Physics analysis can be performed directly on the trigger output with the same online-offline performance.

\section{References}

[1] Alves Jr A A et al. (LHCb collaboration), The LHCb detector at the LHC, 2008 JINST 3 S08005.

[2] Aaij R et al. (LHCb collaboration), LHCb detector performance, 2015 Int. J. Mod. Phys. A30 1530022 [hep-ex/1412.6352].

[3] Aaij R et al. (LHCb collaboration), Performance of the LHCb Vertex Locator, 2014 JINST 9 P09007 [hep-ex/1405.7808]. 
[4] Beringer J et al. (Particle Data Group), Review of Particle Physics, 2012 Phys.Rev. D/ 86 $\mathrm{P} 010001$.

[5] Arink R et al., Performance of the LHCb Outer Tracker, 2014 JINST 9 P01002 [hep-ex/1311.3893].

[6] Adinolfi M et al., Performance of the LHCb RICH detector at the LHC, 2013 Eur. Phys. J. C73 2431 [hep-ex/1211.6759].

[7] Alves Jr A A et al., Performance of the LHCb muon system, 2013 JINST 8 P02022 [hep-ex/1211.1346].

[8] Aaij R et al., The LHCb trigger and its performance in 2011, 2013 JINST 8 P04022 [hep-ex/1211.3055].

[9] Aaij R et al. (LHCb collaboration), Measurement of b-hadron branching fractions for twobody decays into charmless charged hadrons, 2012 JHEP 10037 [hep-ex/1206.2794].

[10] Benson S et al., The LHCb Turbo Stream, in proceedings of CHEP2015, (2015).

[11] Hulsbergen W, The global covariance matrix of tracks fitted with a Kalman filter and an application in detector alignment, 2009 Nucl. Instrum. Meth. A 600471 - 477 [hep-ex/0810.2241].

[12] Amoraal J et al., Application of vertex and mass constraints in track-based alignment, 2013 Nucl. Instrum. Meth. A 71248 - 55 [hep-ex/1207 . 4756] .

[13] Sobel I, An isotropic $3 \times 3$ image gradient operator, 1990 Machine Vision for ThreeDimensional Scenes ed Freeman H (Academic Press) pp 376 - 379. 